\title{
Combined optical and electrical stimulation of neural tissue in vivo
}

\author{
Austin R. Duke, Jonathan M. Cayce, \\ Jonathan D. Malphrus, ${ }^{a}$ Peter Konrad, ${ }^{a, b}$ \\ Anita Mahadevan-Jansen, ${ }^{a, b}$ and E. Duco Jansen ${ }^{a, b, *}$ \\ ${ }^{a}$ Vanderbilt University, Department of Biomedical \\ Engineering, VU Station B, Box 351631, Nashville, \\ Tennessee 37235 \\ ${ }^{b}$ Vanderbilt University, Department of Neurological Surgery, \\ Nashville, Tennessee 37235
}

\begin{abstract}
Low-intensity, pulsed infrared light provides a novel nerve stimulation modality that avoids the limitations of traditional electrical methods such as necessity of contact, presence of a stimulation artifact, and relatively poor spatial precision. Infrared neural stimulation (INS) is, however, limited by a 2:1 ratio of threshold radiant exposures for damage to that for stimulation. We have shown that this ratio is increased to nearly $6: 1$ by combining the infrared pulse with a subthreshold electrical stimulus. Our results indicate a nonlinear relationship between the subthreshold depolarizing electrical stimulus and additional optical energy required to reach stimulation threshold. The change in optical threshold decreases linearly as the delay between the electrical and optical pulses is increased. We have shown that the high spatial precision of INS is maintained for this combined stimulation modality. Results of this study will facilitate the development of applications for infrared neural stimulation, as well as target the efforts to uncover the mechanism by which infrared light activates neural tissue. ( 2009 Society of Photo-Optical Instrumentation Engineers. [DOI: 10.1117/1.3257230]
\end{abstract}

Keywords: infrared neural stimulation; electrical stimulation; compound action potential; latent addition; sciatic nerve.

Paper 09250LR received Jun. 15, 2009; revised manuscript received Aug. 13, 2009; accepted for publication Sep. 8, 2009; published online Nov. 10, 2009.

\section{Introduction}

Recent research demonstrating the feasibility and advantages of stimulating neural tissue with infrared light has generated significant interest. Applications of infrared neural stimulation (INS) range from stimulation of the auditory system for improved cochlear implants to cavernous nerve mapping during prostate resections. ${ }^{1-4}$ While electrical stimulation has long been the method of choice for stimulating neural activity, INS is a capable alternative that provides a contact-free, artifactfree, and spatially precise neural stimulation modality., However, INS is limited by a narrow window for safe stimulation. Wells et al. have shown that radiant exposures $\left(\mathrm{J} / \mathrm{cm}^{2}\right)$ generating laser-induced thermal damage are only a factor of 2 greater than those needed for stimulation. ${ }^{6}$ For INS to be applied at higher repetition rates or radiant exposures much

*Tel: 615-343-1911; Fax: 615-343-7919; E-mail: duco.jansen@vanderbilt.edu

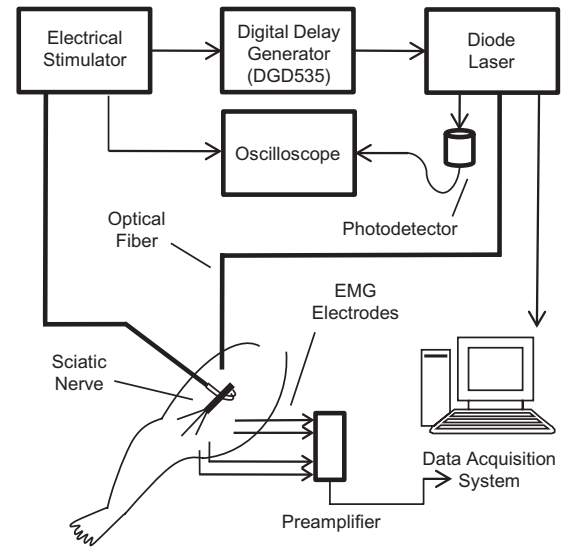

Fig. 1 Schematic representation of the experimental setup used for all experiments in this study.

greater than threshold, the range of radiant exposures for safe yet effective stimulation must be extended. Additionally, implantable INS stimulators may be limited by the laser power necessary for stimulation. Reducing the power requirements of the INS stimulator will facilitate the translation of INS technology into an implantable device.

We hypothesize that the nerve excitability to INS may be enhanced by applying a subthreshold electrical stimulus concomitantly with the delivery of pulsed infrared light, thus lowering the threshold for optical stimulation while maintaining spatial precision and mitigating the risk of laser-induced tissue damage. To test this hypothesis, we varied the magnitude of the subthreshold electrical stimulus to determine the relationship between the electrical stimulus magnitude and the requisite amount of optical energy to achieve stimulation. We then investigated the delay of the infrared pulse relative to the electrical pulse to determine the optimal pulse synchronization for minimizing the optical energy required. By reducing the threshold radiant exposure of infrared light needed to achieve stimulation by nearly threefold, we have greatly increased the safe and effective range of INS. Last, it was confirmed that the spatial precision of INS is maintained for this combined optical and electrical stimulation modality.

\section{Methods}

Male Sprague-Dawley rats (300 to $400 \mathrm{~g}$ ) were anesthetized with $50 \%$ urethane $(1.5 \mathrm{~g} / \mathrm{kg}$ IP), and the sciatic nerve was exposed from the pelvic cavity to the knee by blunt dissection. An average of 3 to 4 measurements were taken from 24 nerves, yielding a total of 92 data points for this study. Saline was continuously applied to prevent dehydration of the nerve.

The system diagram used for these experiments is shown in Fig. 1. An electrical stimulator (Grass S44; Grass Medical Instruments, Quincy, Massachusetts) was connected to a bipolar hook electrode placed under the main trunk of the sciatic nerve. A pulsed infrared diode laser $(\lambda=1.875 \mu \mathrm{m}$; Lockheed Martin Aculight Capella) was coupled to a $400-\mu$ m-diam optical fiber (Ocean Optics). The distal end of the fiber was positioned directly above the nerve and approximately $700 \mu \mathrm{m}$ from the surface of the nerve, in the same location as

1083-3668/2009/14(6)/060501/3/\$25.00 @ 2009 SPIE 
the electrode. Using the knife edge technique, ${ }^{7}$ the laser spot size on the nerve was determined to be $0.3584 \mathrm{~mm}^{2}$. The optical penetration depth in tissue at the selected wavelength was approximately $400 \mu \mathrm{m}$, which has been shown to allow selective recruitment of a single 50- 200- $\mu \mathrm{m}$-diam fascicle positioned below the $200-\mu \mathrm{m}$-thick perineurium. ${ }^{4}$ The pulse duration for both electrical and optical stimulation was $2 \mathrm{~ms}$, which was dictated by the minimum pulse duration needed to obtain sufficient pulse energy to optically stimulate the nerve. Pulses were delivered at a repetition rate of $2 \mathrm{~Hz}$ for all experiments. Monophasic electrical stimulation was used with stimulation threshold voltages averaging $0.86 \pm 0.30 \mathrm{~V}$. The electrical stimulator and the diode laser were synchronized by a digital delay generator (Stanford Research Systems, DGD535). The Nicolet Endeavor Evoked Potentials System was used for electrophysiological evaluations. Needle electrodes were inserted into the biceps femoris and gastrocnemius in a bipolar configuration.

Electrical and optical pulses were first delivered simultaneously. For all experiments, stimulation threshold is defined as the minimum induced current (electrical stimulation) and/or radiant exposure (INS) needed to induce a sustained visible compound muscle action potential (CMAP) in response to stimulation. After finding the electrical stimulation threshold, the electrical stimulus was reduced to a known amount (i.e., 90\% of threshold). An optical stimulus was then applied concomitantly with the electrical stimulus, and its magnitude was increased until reaching threshold. The electrical stimulus was removed, and the stimulation threshold was found using only INS. This process was repeated to establish a relationship describing the relative amounts of electrical and optical energies needed to reach the threshold. In a second experiment, electrical stimulation was set to $90 \%$ of threshold. Using the digital delay generator, the arrival of the optical stimulus was delayed relative to the electrical stimulus, and the amount of additional optical energy needed to achieve stimulation was determined.

\section{Results}

Figure 2(a) demonstrates the effects of combining electrical and optical stimulation. Data points reflect the amount of optical energy (\% INS threshold) required to reach the stimulation threshold when applied concurrently with an electrical stimulus (\% electrical stimulation threshold). The best-fit line models the data incorporating the known endpoints where $100 \%$ of either modality alone is required to reach the stimulation threshold. Interestingly, the data do not fit a linear relationship. Rather, the required optical energy can be predicted by a logarithmic relationship:

$$
O=0.22 \ln (1-E)+1
$$

with $R^{2}=0.56$, where $O$ is the optical energy (\% INS threshold), and $E$ is the magnitude of the electrical stimulus (\% electrical stimulation threshold). The data in Fig. 2 show significant variance that can be attributed to inter- and intraanimal variability; limitations of the experimental setup - in particular, the spatial localization of the stimulation electrodes and fiber optic; and the fact that near the electrical stimulation threshold (the steep part of the curve), minor fluctuations in
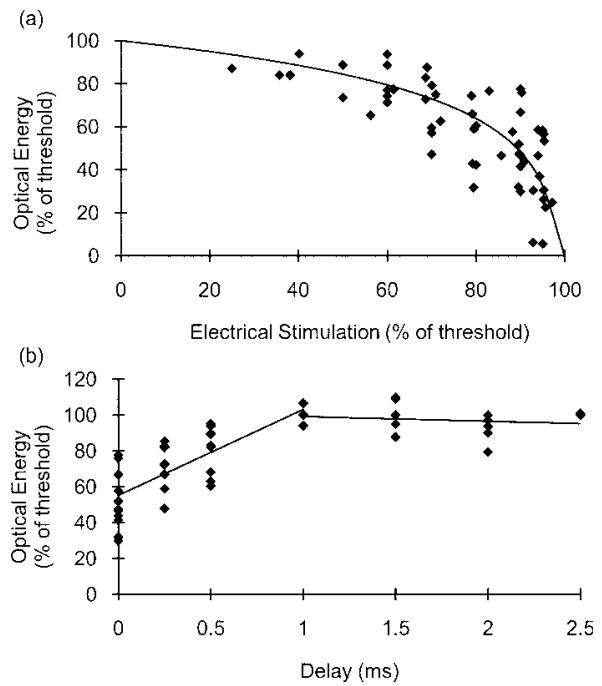

Fig. 2 Results of combining optical stimulation with electrical stimulation. (a) Optical energy (\% of threshold) required to reach stimulation threshold as a function of subthreshold electrical stimulus; and (b) optical energy (\% of threshold) required to reach stimulation threshold as a function of delay between electrical ( $90 \%$ of threshold) and optical stimuli.

electrical stimulation may result in significant changes in optical energy required.

If the electrical stimulus is applied at $95 \%$ of the electric threshold, then the optical threshold will be reduced by a factor of nearly 3 according to Eq. (1). For $80 \%$ or $90 \%$ of threshold, the optical threshold is reduced by 1.54 -fold and 2.03 -fold, respectively. This reduction in optical threshold significantly increases the window for safe INS, as less energy is required to stimulate, thereby reducing the heat load in the tissue. If the ratio of damage threshold to stimulation threshold for INS alone is assumed to be 2:1, as reported by Wells et al., ${ }^{6}$ we can predict that applying an electrical stimulus at $90 \%$ of electrical stimulation with INS will increase this ratio to 4.05:1. For electrical stimuli at $80 \%$ and $95 \%$, the ratio is predicted to be approximately $3.10: 1$ and 5.87:1, respectively. Threshold radiant exposures for INS alone averaged $1.69 \pm 0.30 \mathrm{~J} / \mathrm{cm}^{2}$. Combined with a subthreshold electrical stimulus, radiant exposures were reduced to $1.49 \pm 0.22 \mathrm{~J} / \mathrm{cm}^{2}$ at $60 \%$ of electrical threshold and $0.60 \pm 0.29 \mathrm{~J} / \mathrm{cm}^{2}$ at $95 \%$ of electrical threshold. While the INS threshold radiant exposures reported here (using a $400-\mu \mathrm{m}$ fiber) are higher than those previously published for the rat sciatic nerve (and above the published radiant exposures for thermal damage) that were obtained using a $600-\mu \mathrm{m}$ fiber, ${ }^{6}$ this can be accounted for by the known fiber diameter dependence of thermal distributions as well as known morphological changes over the length of the nerve. In addition, there are several subtle differences in the laser parameters and endpoint definition between the current and previously reported results. Thus, a direct comparison between these absolute values should be made with caution. No visible indication of thermal damage was present at the radiant exposures used in the current study.

Figure 2(b) demonstrates the effects of delaying the optical stimulus relative to the electrical stimulus. The results indicate that the greatest benefit is achieved when the pulses are de- 


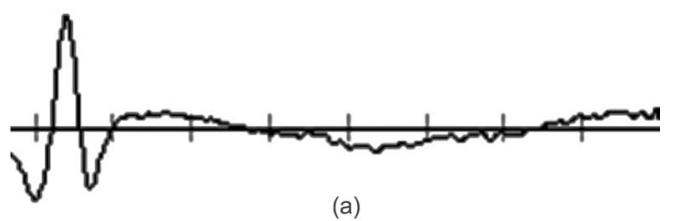

$20 \mu \mathrm{V} \underbrace{}_{2 \mathrm{~ms}}$

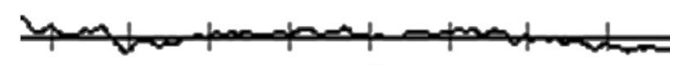

(b)

Fig. 3 Spatial selectivity is maintained with combined optical and electrical stimulation. (a) Average of 20 consecutive recordings of CMAPs from electrodes placed in biceps femoris; and (b) the same recordings as (a) for electrodes placed in gastrocnemius.

livered simultaneously. For delays up to $1 \mathrm{~ms}$, the radiant exposure necessary for stimulation appears to increase linearly. For delay times $>1 \mathrm{~ms}$, there are no benefits of combining the modalities as $100 \%$ of the optical threshold is needed to achieve stimulation. Figure 3 illustrates that the spatial selectivity of INS is preserved in this combined stimulation modality. Note how the stimulated CMAP is present in only one muscle group.

\section{Discussion and Conclusions}

The results of this study confirm the hypothesis that delivery of a subthreshold electrical stimulus concurrently with INS will lower the required optical energy per pulse to achieve stimulation and thus demonstrate proof of concept for a combined electrical/optical nerve stimulator. This suggests that the ratio of safe to damaging radiant exposures of INS may be increased by the simultaneous delivery of a subthreshold electrical stimulus. These findings are practical for the further developments of INS technology and may also shed light on the underlying mechanism of INS. A significant aspect of these results is that the reduction of INS threshold for a given subthreshold electrical stimulus does not follow a linear trend [Fig. 2(a)]. This implies that electrical stimulation and INS do not function by the same mechanism. Otherwise, one would expect that a linear superposition would achieve stimulation, as is seen when two simultaneous electrical pulses are combined. $^{8}$

Delivering a subthreshold electrical stimulus to enhance the excitability of neural tissue to an added electrical stimulus is not a foreign concept. ${ }^{8-10}$ The mechanism by which threshold changes occur as a result of a subthreshold stimulus has been explained by a mathematical model of induced ionic currents with enhanced excitability primarily following membrane potential. Persistent and transient $\mathrm{Na}^{+}$currents initiate "superexcitability;" $\mathrm{Na}^{+}$channel inactivation, decay in the leakage current, and activation of outward $\mathrm{K}^{+}$currents (primarily slow $\mathrm{K}^{+}$channels) cause the decline in excitability over time. ${ }^{10,11}$

While the results of this study are encouraging and highlight the advantages of a combined optical and electrical stimulation modality, a better fundamental understanding of this new stimulation paradigm is necessary for further devel- opment. Primarily, it must be determined whether a combination of a subthreshold electrical stimulus with a subthreshold optical stimulus will result in less tissue damage than INS alone. The effects of laser-induced tissue damage from INS are well characterized, but the damaging effects of electrical stimulation are not as clear-especially in the context of a combined stimulation modality. ${ }^{6}$ It remains to be determined whether there exists an optimal combination of optical and electrical stimulation parameters which will minimize tissue damage.

Our data emphasize the obvious and practical benefits of combined optical and electrical stimulation. Further evaluation of the available parameters is necessary, but the proof of concept is evident. The subthreshold electrical stimulus clearly reduces the potential risks of laser-induced damage without interfering with the spatial precision inherent to INS (Fig. 3). These results will facilitate the development of implantable INS stimulators by reducing required laser power, as well as benefit researchers needing a safe, spatially precise stimulation modality. While the benefits of applying INS concurrently with electrical stimulation suggest that ionic currents contribute to the mechanism of INS, the differences in excitability between delayed optical and electrical stimuli following an initial subthreshold depolarizing stimulus indicate that the mechanism of INS is more involved.

\section{Acknowledgments}

This work was funded by the NIH (R01 NS052407-01).

\section{References}

1. N. M. Fried, G. A. Lagoda, N. J. Scott, L. M. Su, and A. L. Burnett, "Noncontact stimulation of the cavernous nerves in the rat prostate using a tunable-wavelength thulium fiber laser," J. Endourol 22(3), 409-413 (2008).

2. A. D. Izzo, C. P. Richter, E. D. Jansen, and J. T. Walsh, "Laser stimulation of the auditory nerve," Lasers Surg. Med. 38(8), 745-753 (2006).

3. J. Wells, C. Kao, E. D. Jansen, P. Konrad, and A. Mahadevan-Jansen, "Application of infrared light for in vivo neural stimulation," $J$. Biomed. Opt. 10(6), 064003 (2005).

4. J. Wells, P. Konrad, C. Kao, E. D. Jansen, and A. Mahadevan-Jansen, "Pulsed laser versus electrical energy for peripheral nerve stimulation," J. Neurosci. Methods 163(2), 326-337 (2007).

5. J. Wells, C. Kao, K. Mariappan, J. Albea, E. D. Jansen, P. Konrad, and A. Mahadevan-Jansen, "Optical stimulation of neural tissue in vivo," Opt. Lett. 30(5), 504-506 (2005).

6. J. D. Wells, S. Thomsen, P. Whitaker, E. D. Jansen, C. C. Kao, P. E. Konrad, and A. Mahadevan-Jansen, "Optically mediated nerve stimulation: identification of injury thresholds," Lasers Surg. Med. 39(6), 513-526 (2007).

7. J. M. Khosrofian and B. A. Garetz, "Measurement of a Gaussian laser-beam diameter through the direct inversion of knife-edge data," Appl. Opt. 22(21), 3406-3410 (1983).

8. H. Bostock and J. C. Rothwell, "Latent addition in motor and sensory fibers of human peripheral nerve," J. Physiol. (London) 498(Pt 1), 277-294 (1997).

9. H. Bostock, C. S. Lin, J. Howells, L. Trevillion, S. Jankelowitz, and D. Burke, "After-effects of near-threshold stimulation in single human motor axons," J. Physiol. (London) 564(Pt 3), 931-940 (2005).

10. D. Burke, J. Howells, L. Trevillion, P. A. McNulty, S. K. Jankelowitz, and M. C. Kiernan, "Threshold behavior of human axons explored using subthreshold perturbations to membrane potential," $J$. Physiol. (London) 587(Pt 2), 491-504 (2009).

11. H. Bostock, M. Baker, P. Grafe, and G. Reid, "Changes in excitability and accommodation of human motor axons following brief periods of ischaemia," J. Physiol. (London) 441, 513-535 (1991). 